Supporting Information for

\title{
Non-Interpenetrated Metal-Chalcogenide Cluster-Based Frameworks Assembled by Small-sized Penta- Supertetrahedral Clusters
}

Chengdong Liu, Zhou Wu, Xiao-Li Wang, Yayun Ding, Jiaxu Zhang, * Weijie Yang, Xiang Wang, Rui Zhou, and Tao Wu

†College of Chemistry, Chemical Engineering and Materials Science, Soochow University, Suzhou, Jiangsu 215123, China.

*E-mail: jxzhang@suda.edu.cn 


\section{Experimental Section}

Powder X-Ray Diffraction (PXRD). PXRD data were collected at room temperature on a desktop diffractometer (D2 PHASER, Bruker, Germany) using $\mathrm{Cu}-\mathrm{K} \alpha(\lambda=$ $1.54184 \AA$ ) radiation operated in the $2 \theta$ range of $5-50^{\circ}$ at $30 \mathrm{kV}$ and $10 \mathrm{~mA}$. The samples were ground before the test.

UV-Vis Absorption Measurement. UV-Vis diffusion reflectance spectra of MCCF20 and MCCF-21 were performed on a spectrophotometer (SHIMADZU UV-3600 UVVis-NIR). The absorption spectra were calculated from diffuse reflectance spectra through using the Kubelka-Munk function: $F(R)=\alpha / S=(1-R)^{2} / 2 R$, where $R$ is the reflection, $\alpha$ is the absorption coefficient, and $\mathrm{S}$ is the scattering coefficient.

Elemental Analysis. Energy dispersive spectroscopy (EDS) analysis were performed on a ZEISS EVO 18 scanning electron microscope (SEM) equipped with energy dispersive spectroscopy detector with accumulation time of $40 \mathrm{~s}$ and acceleration voltage of $25 \mathrm{kV}$. Elemental analysis of C, $\mathrm{H}$ and $\mathrm{N}$ was performed on VARIO EL III elemental analyzer.

Thermogravimetric Analysis (TGA). TGA measurements were performed with a Shimadzu TGA-50 system under nitrogen flow. The TG curves were performed by heating the sample from room temperature to $800^{\circ} \mathrm{C}$ with heating rate of $10^{\circ} \mathrm{C} / \mathrm{min}$.

Ion Exchange. The samples of MCCF-20 (20 mg) and MCCF-21 (20 mg) were immersed in $20 \mathrm{~mL}$ aqueous solution of $\mathrm{CsCl}(1 \mathrm{M})$ in glass vials at room temperature. During the treatment, the $\mathrm{CsCl}$ solution $(1 \mathrm{M})$ was refreshed twice. After 12 hours, the crystals were filtered out from the solution and washed with water several times to remove the residual $\mathrm{Cs}^{+}$ions adsorbed on the surface of the crystals. 

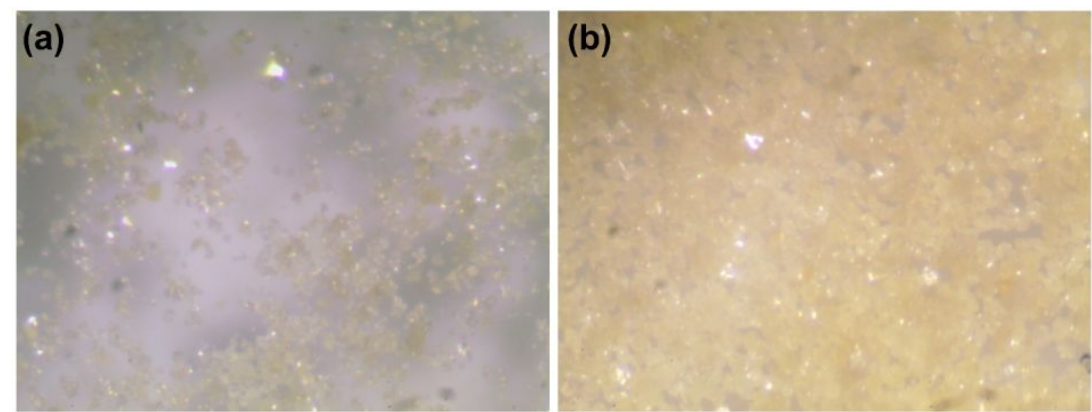

Figure S1. Optical images of as-synthesized (a) MCCF-20 and (b) MCCF-21.

Table S1. The detailed information of synthetic conditions for MCCF-20 and MCCF21.

\begin{tabular}{c|ccc|ccc}
\hline Conditions & \multicolumn{3}{|c|}{ Ratio of Zn to Sn $^{\mathbf{a}}$} & \multicolumn{3}{c}{ Temperature $^{\mathbf{b}}$} \\
\hline Value & $2: 1$ & $1: 1$ & $1: 2$ & $<165^{\circ} \mathrm{C}$ & $170^{\circ} \mathrm{C}$ & $>175^{\circ} \mathrm{C}$ \\
Product & Yes & Yes & Yes & No & Yes & No \\
Purity & impure & pure & impure & No & pure & No \\
\hline
\end{tabular}

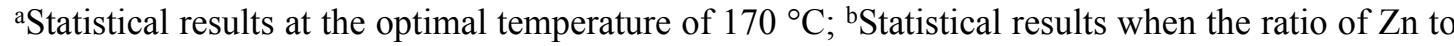
Sn is $1: 1$.
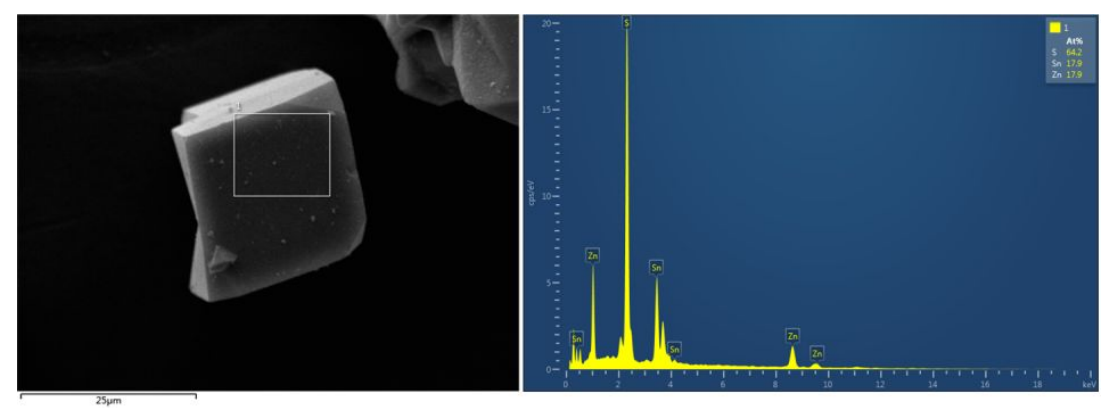

Figure S2. Left: SEM image of as-synthesized MCCF-20. Right: EDS of MCCF-20. The results clearly confirmed the presence of $\mathrm{Zn}$, Sn and S elements, and the measured molar ratio of $\mathrm{Zn} / \mathrm{Sn} / \mathrm{S}$ is $1: 1: 3.59$, which is consistent with the theoretical value of 1 : $1: 3.75$. 

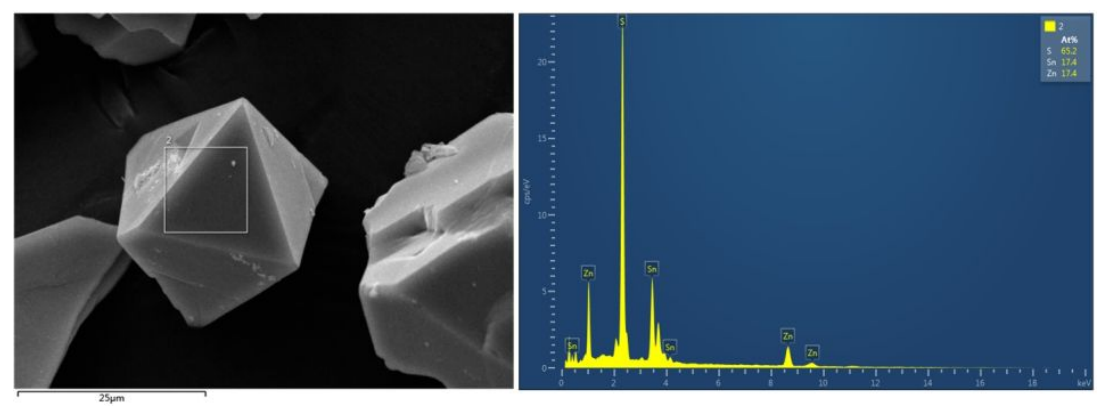

Figure S3. Left: SEM image of as-synthesized MCCF-21. Right: EDS of MCCF-21. The results clearly confirmed the presence of $\mathrm{Zn}$, Sn and S elements, and the measured molar ratio of $\mathrm{Zn} / \mathrm{Sn} / \mathrm{S}$ is $1: 1: 3.75$, which is consistent with the theoretical value of 1 : $1: 3.75$.

(a)

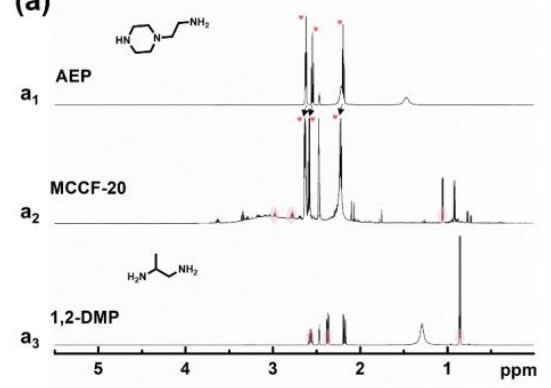

(b)

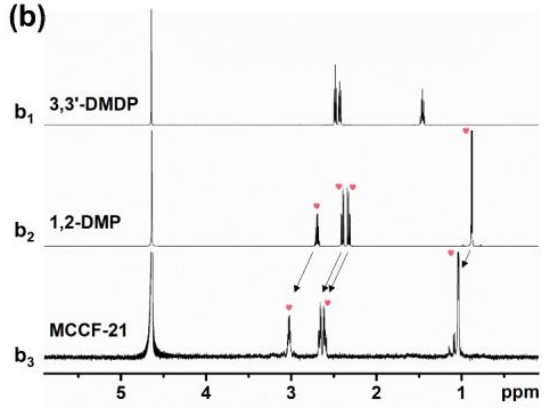

Figure S4. ${ }^{1} \mathrm{H}$ NMR spectra (600 MHz, DMSO- $d_{6}$ (a1-a3), $\mathrm{D}_{2} \mathrm{O}$ (b1-b3), 298K) of (a1) AEP, (a2) MCCF-20, (a3 and b2) 1,2-DMP, (b1) 3,3'-DMDP and (b3) MCCF-21. The presence of protonated AEP and 1,2-DMP are demonstrated in MCCF-20. For MCCF21, the signal of 1,2-DMP is observed, whereas no signal of 3,3'-DMDP is observed.

Table S2. EA results of MCCF-20 and MCCF-21.

\begin{tabular}{|c|c|c|c|c|}
\hline & Elements (wt.) & N (\%) & $\mathrm{C}(\%)$ & H (\%) \\
\hline \multirow{3}{*}{ MCCF-20 } & Experimental-1 & 12.21 & 20.17 & 4.82 \\
\hline & Experimental-2 & 12.09 & 19.98 & 4.94 \\
\hline & Calculated & 12.17 & 20.13 & 4.69 \\
\hline \multirow{3}{*}{ MCCF-21 } & Experimental-1 & 10.17 & 13.04 & 4.19 \\
\hline & Experimental-2 & 9.95 & 12.87 & 3.92 \\
\hline & Calculated & 10.07 & 12.96 & 3.99 \\
\hline
\end{tabular}

Based on the synthesis conditions, the results of ${ }^{1} H N M R$ spectra, SC-XRD and EA, the formula of MCCF-20 and MCCF-21 are determined to be $(H-1,2-D M P)_{1.2} \cdot(H-$ $A E P)_{4.8} \cdot\left[Z_{4} S_{4} S_{15}\right]$ and $(H-1,2-D M P)_{6} \cdot\left[Z_{4} S_{4} n_{15}\right]$, respectively. 


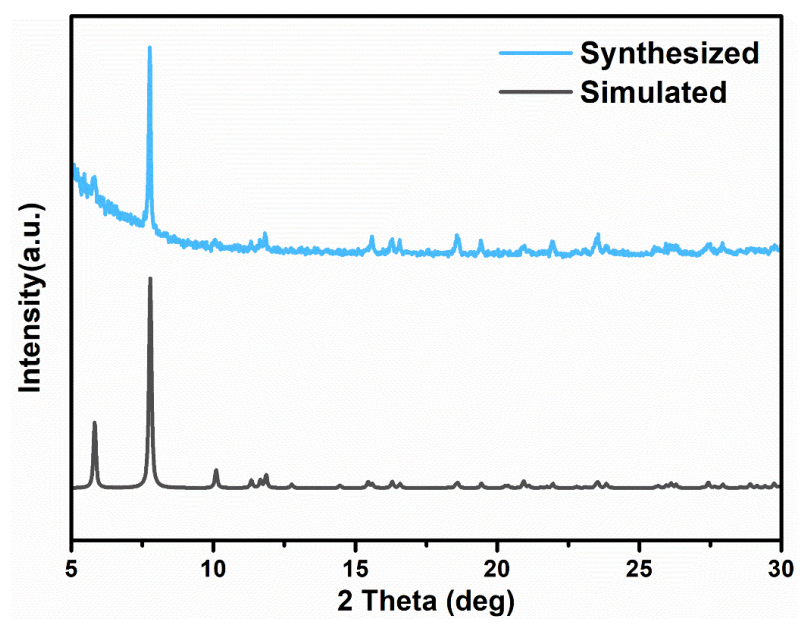

Figure S5. The simulated and experimental PXRD patterns of MCCF-20.

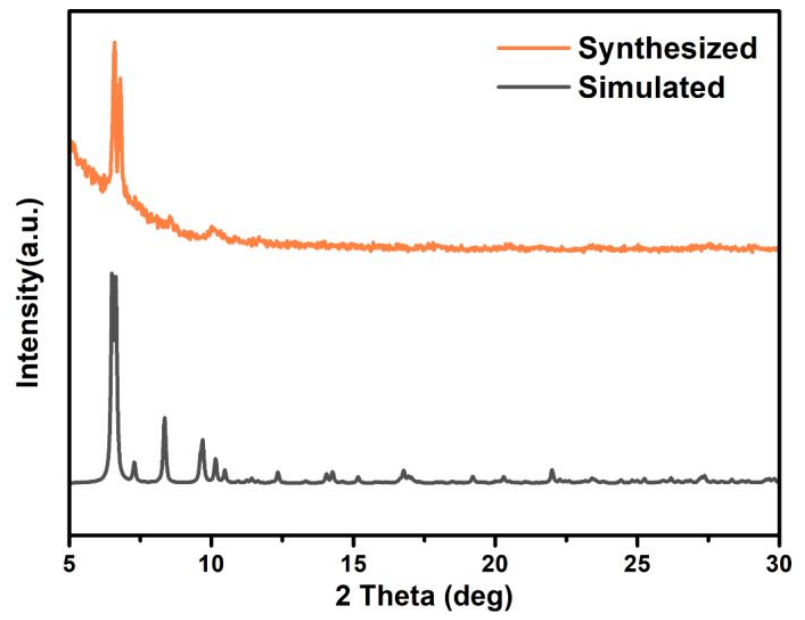

Figure S6. The simulated and experimental PXRD patterns of MCCF-21.

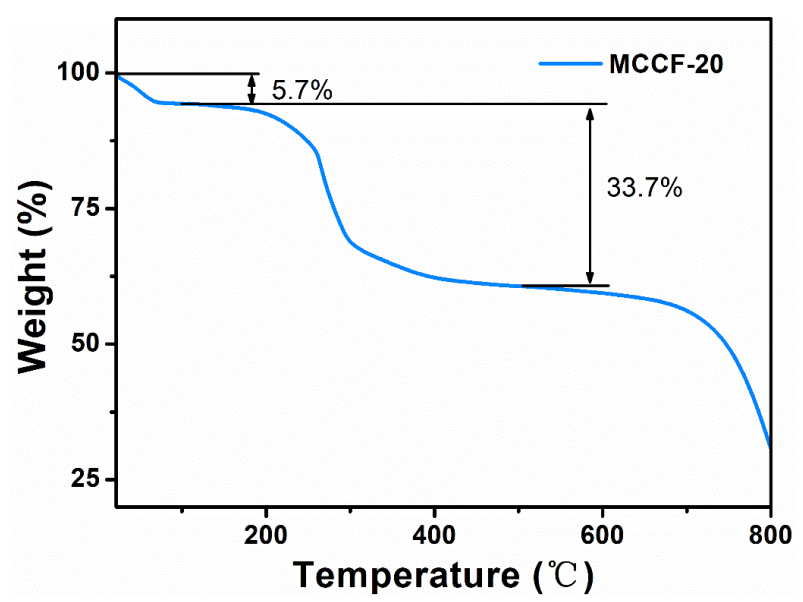

Figure S7. TG curves of MCCF-20. The initial gradual weight loss of 5.7\% between 25-100 ${ }^{\circ} \mathrm{C}$ could be attributed to loss of some structure directing agents and solvent molecules. A further abrupt weight loss of $33.7 \%$ between $200-450^{\circ} \mathrm{C}$ are attributed to 
the carbonization of most structure directing agents (1,2-DMP and AEP).

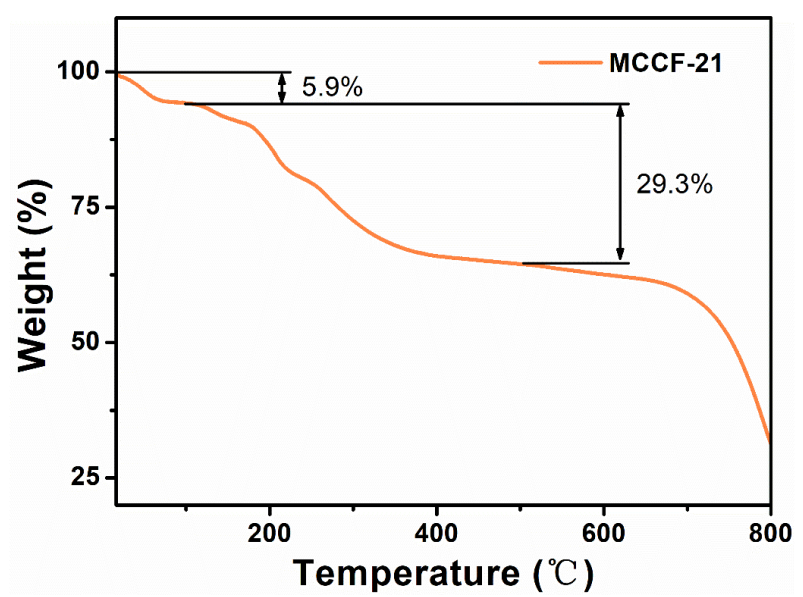

Figure S8. TG curves of MCCF-21. The initial gradual weight loss of 5.9\% between $25-100{ }^{\circ} \mathrm{C}$ could be attributed to loss of some structure directing agents and solvent molecules. A further abrupt weight loss of $29.3 \%$ between $200-450^{\circ} \mathrm{C}$ are attributed to the carbonization of most structure directing agents (1,2-DMP).

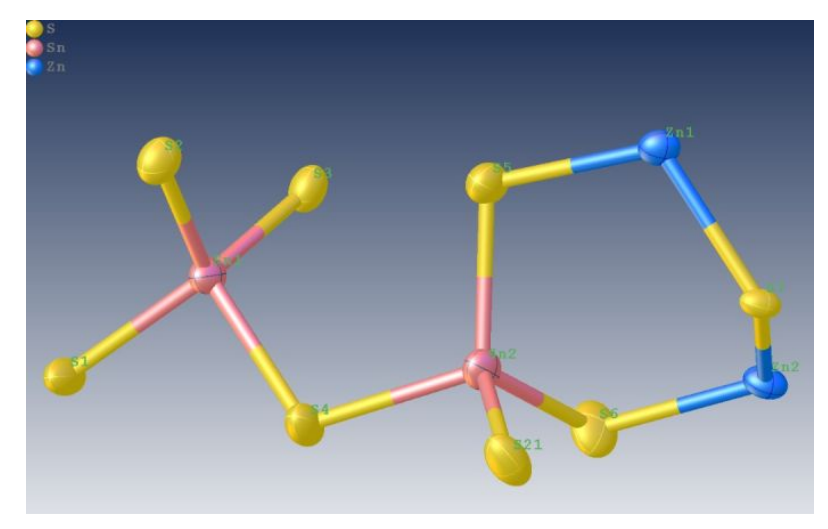

Figure S9. The asymmetric unit in the framework of $\mathbf{M C C F - 2 0} \mathbf{L}$.
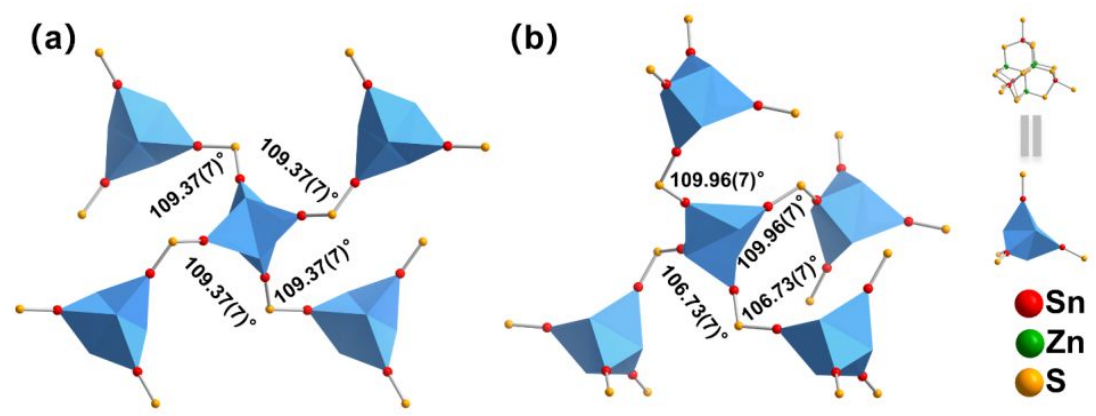

Figure S10. Each P1 cluster connects to four adjacent P1 clusters, as observed in (a) MCCF-20 ${ }_{\mathrm{L}}$ and (b) MCCF-21. 


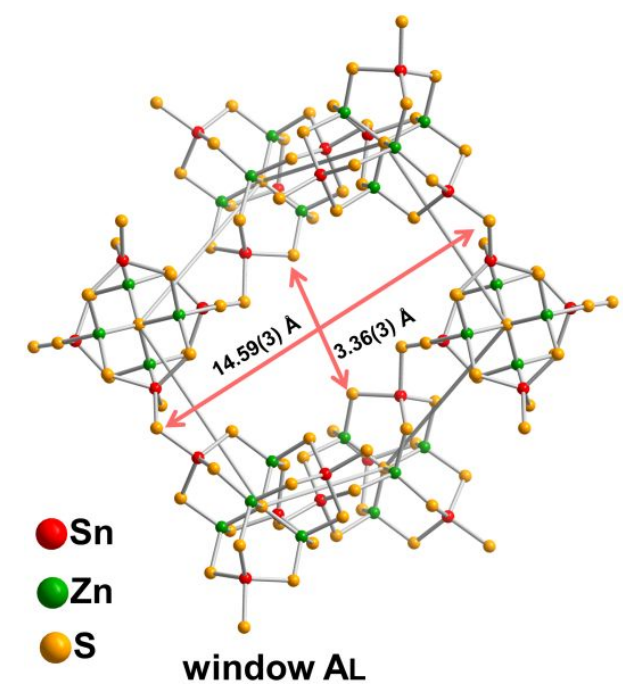

Figure S11. 6-MR window $A_{L}$ with the aperture sizes of $14.59(3) \times 3.36(3) \AA$ in MCCF-20 . .

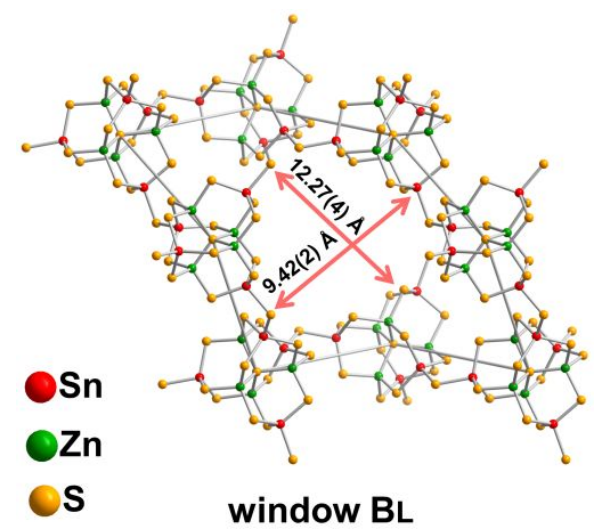

Figure S12. 8-MR window $B_{L}$ with the aperture sizes of $12.27(4) \times 9.42(2) \AA$ in $\mathrm{MCCF}-20_{\mathrm{L}}$.

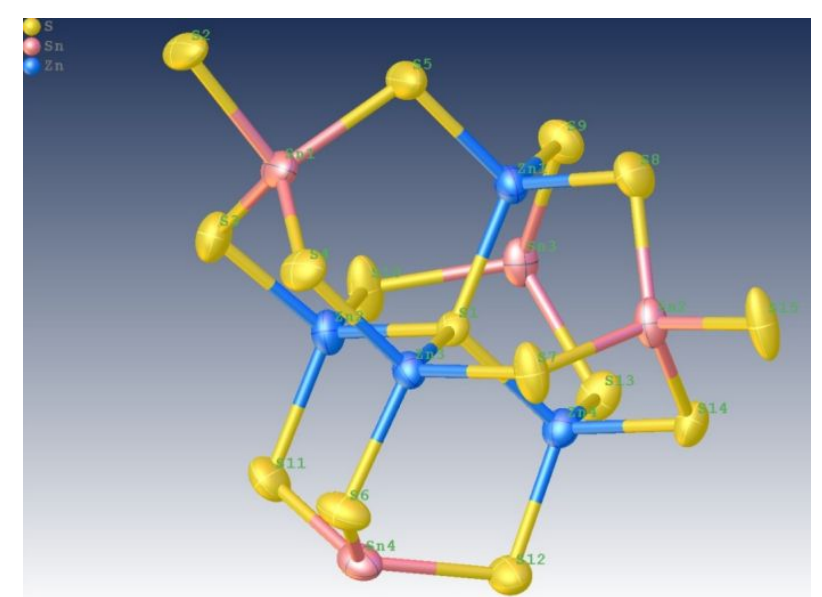

Figure S13. The asymmetric unit in the framework of MCCF-21. 


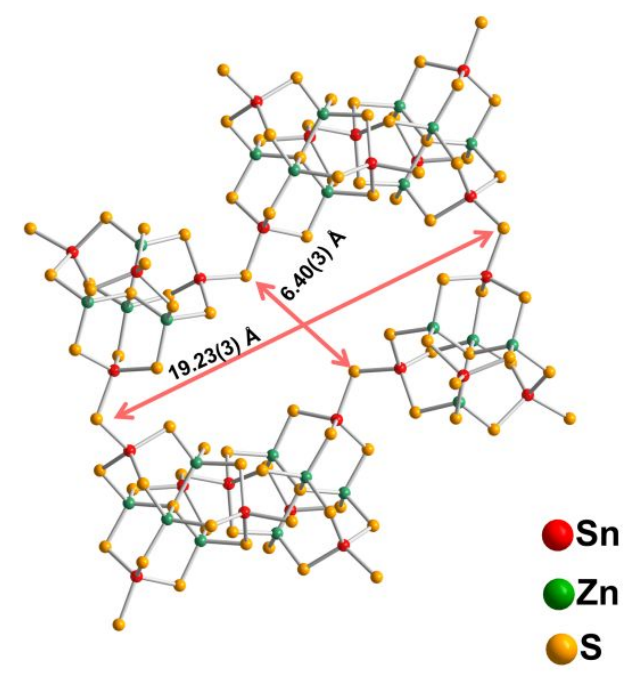

window C

Figure S14. 6-MR window $C$ with the aperture sizes of $19.23(3) \times 6.40(3) \AA$ in MCCF21.

Table S3. Comparison of MCCF-20/MCCF-21 with other open frameworks based on $\mathrm{P} 1-\mathrm{ZnSnS}$

\begin{tabular}{cccc}
\hline Compounds & Formula & Topology & Structure-directing agent \\
\hline MCCF-20 & {$\left[\mathrm{Zn}_{4} \mathrm{Sn}_{4} \mathrm{~S}_{15}\right]^{6-}$} & Quartz & $(\mathrm{H}-1,2-\mathrm{DMP}),(\mathrm{H}-\mathrm{AEP})$ \\
MCCF-21 & {$\left[\mathrm{Zn}_{4} \mathrm{Sn}_{4} \mathrm{~S}_{15}\right]^{6-}$} & Diamond & $(\mathrm{H}-1,2-\mathrm{DMP}),(\mathrm{H}-3,3$ '-DMDP) \\
${ }^{1}$ Compound 1 & {$\left[\mathrm{Zn}_{4} \mathrm{Sn}_{5} \mathrm{~S}_{17}\right]^{6-}$} & Diamond & $\mathrm{K}^{+}$ \\
${ }^{1}$ Compound 2 & {$\left[\mathrm{Zn}_{4} \mathrm{Sn}_{5} \mathrm{~S}_{17}\right]^{6-}$} & Diamond & $\mathrm{K}^{+}, \mathrm{Cs}^{+}$ \\
${ }^{1}$ Compound 3 & {$\left[\mathrm{Zn}_{4} \mathrm{Sn}_{5} \mathrm{~S}_{17}\right]^{6-}$} & Diamond & $\mathrm{K}^{+}, \mathrm{Rb}^{+}$ \\
\hline
\end{tabular}

(1) Angew. Chem. Int. Ed. 2005, 44, 3552-3555
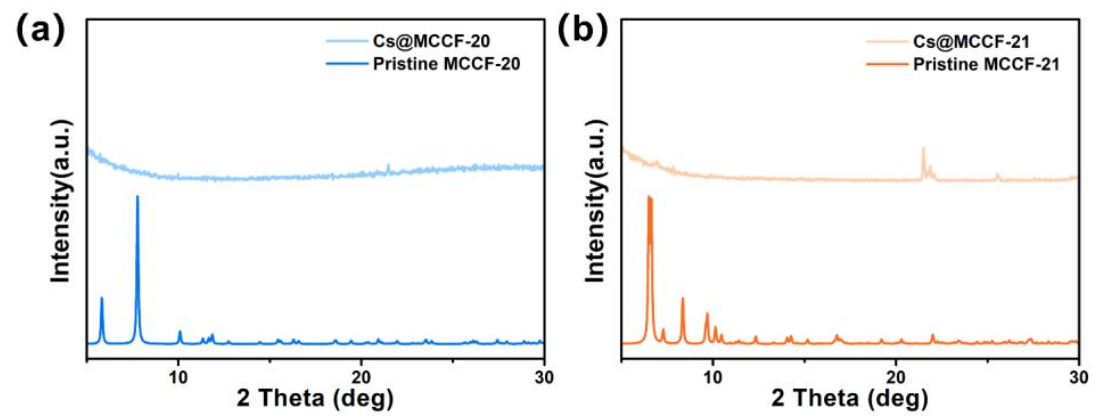

Figure S15. (a) PXRD patterns of pristine MCCF-20 and Cs@MCCF-20. (b) PXRD patterns of pristine MCCF-21 and Cs@MCCF-21. 


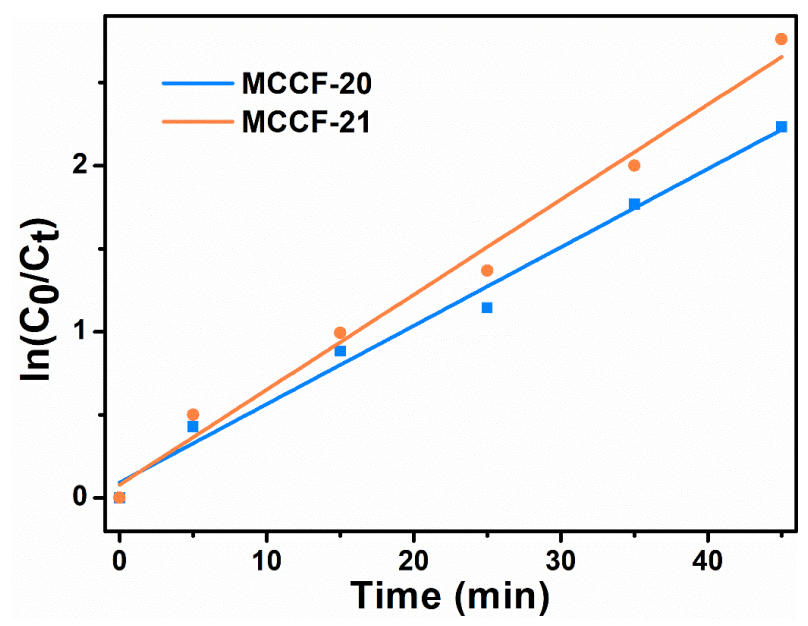

Figure S16. The calculated degradation rate of MB over MCCF-20 and MCCF-21.

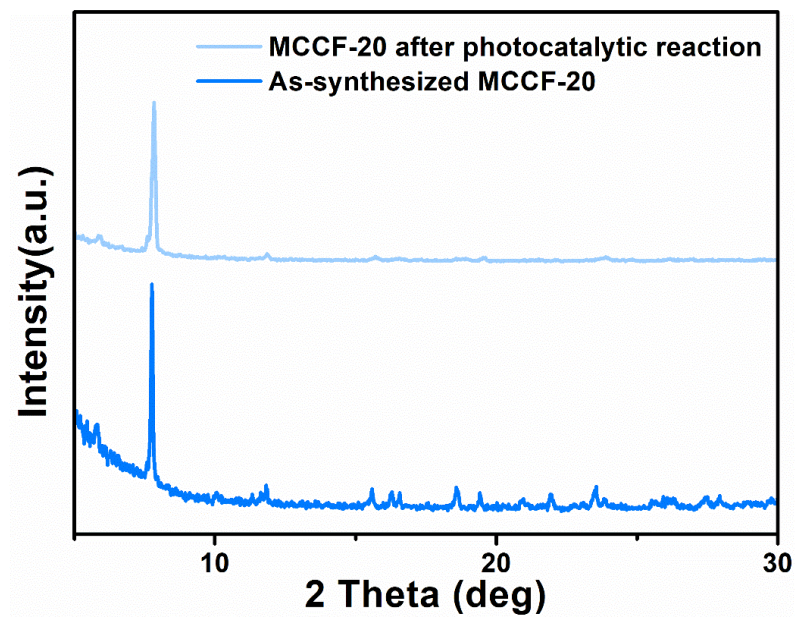

Figure S17. PXRD patterns of MCCF-20 before and after photocatalytic reactions.

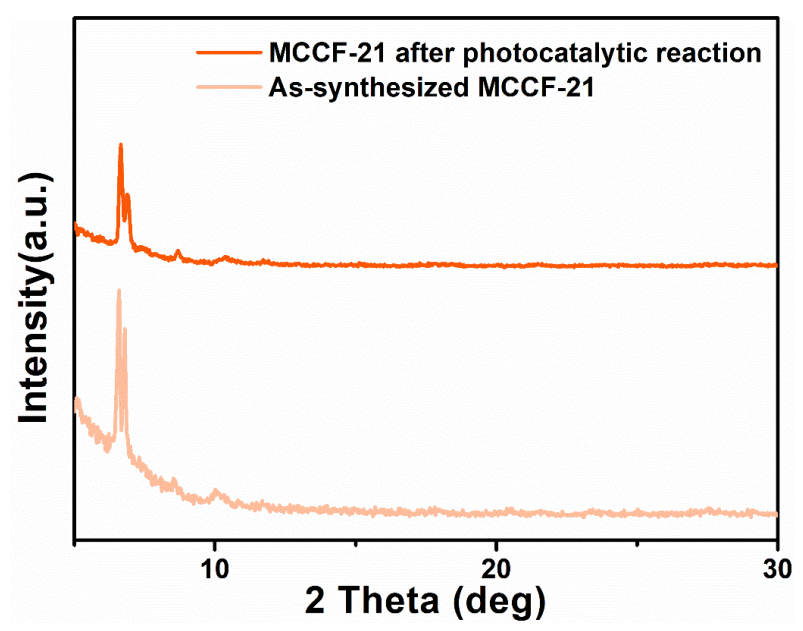

Figure S18. PXRD patterns of MCCF-21 before after photocatalytic reactions. 

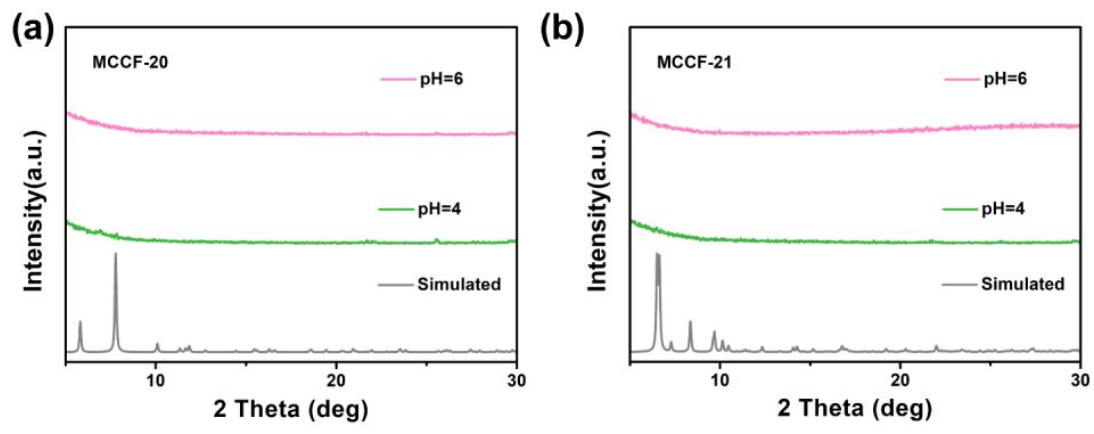

Figure S19. PXRD patterns of (a) MCCF-20 and (b) MCCF-21 after immersion in acidic solutions.
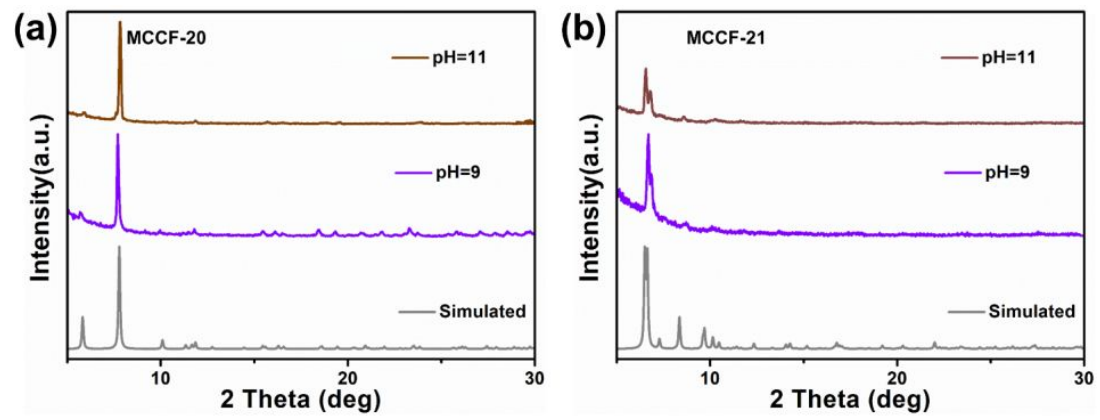

Figure S20. PXRD patterns of (a) MCCF-20 and (b) MCCF-21 after immersion in aqueous alkaline solutions with different $\mathrm{pH}$ values.
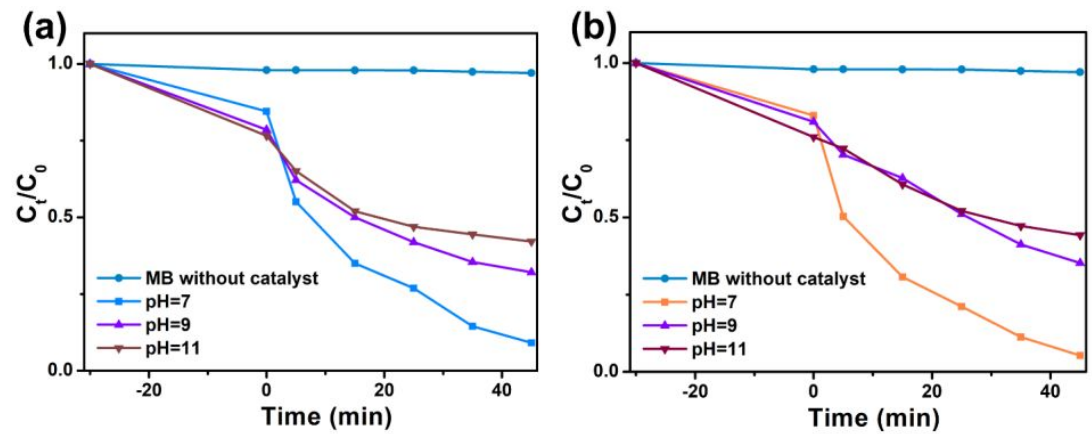

Figure S21. Photocatalytic activity of (a) MCCF-20 and (b) MCCF-21 in different pH values as indicated by $\mathrm{MB}$ concentration versus irradiation time. 
Table S4. Summary of crystalline metal chalcogenides as photocatalysts for organic pollutant degradation.

\begin{tabular}{|c|c|c|c|c|c|c|}
\hline Compound $^{\mathrm{a}}$ & Interpenetration & $\begin{array}{l}\text { Catalyst } \\
(\mathrm{mg} / \mathrm{mL})\end{array}$ & $\begin{array}{l}\text { Pollutants }\left(\times 10^{-}\right. \\
\left.{ }^{5} \mathbf{M}\right)^{b}\end{array}$ & $\begin{array}{c}\text { Completiontime } \\
\text { (min) and Ratio (\%) }\end{array}$ & $\begin{array}{c}k c \\
\left(\min ^{-1}\right)\end{array}$ & $\begin{array}{c}\text { Photocurren } \\
\qquad\left(\mu \mathrm{A} \mathrm{cm}^{-2}\right)\end{array}$ \\
\hline MCCF-20 This work & No & 2 & MB, 1.9 & $45,>90$ & 0.047 & 1.05 \\
\hline MCCF-21 ${ }^{\text {This work }}$ & No & 2 & MB, 1.9 & $45,>94$ & 0.057 & 2.84 \\
\hline$\left[\mathrm{Cu}_{5} \mathrm{Sn}_{4} \mathrm{GaSe}_{14}\right]^{2}$ & No & 0.5 & $\mathrm{MB}, 1.8$ & 80,99 & 0.0917 & 0.720 \\
\hline$\left[\mathrm{In}_{42.6} \mathrm{Sn}_{7.4} \mathrm{~S}_{90}\right]^{3}$ & No & 0.5 & $\mathrm{MB}, 0.05$ & 60,95 & 0.05 & NA \\
\hline$\left[\operatorname{In}_{36} \mathrm{Se}_{69}\right]^{4}$ & No & 2 & $\mathrm{RhB}, 8$ & $240,>90$ & NA & NA \\
\hline$\left[\mathrm{Ag}_{4} \mathrm{Sn}_{3} \mathrm{~S}_{8}\right]^{5}$ & No & 0.6 & $\mathrm{CV}$ & 200,92 & NA & NA \\
\hline$\left[\mathrm{Sn}_{20} \mathrm{O}_{10} \mathrm{~S}_{34}\right]^{6}$ & Yes & 0.6 & $\mathrm{RhB}, 20.2, \mathrm{pH}=1$ & $270,>98$ & NA & 0.58 \\
\hline$\left[\mathrm{Cd}_{8} \mathrm{~S}(\mathrm{SPh})_{14}(\mathrm{bpy})\right]^{7}$ & $1 \mathrm{D}$ & 20 & $\mathrm{MB}, 4.18$ & 180,95 & NA & NA \\
\hline$\left[\mathrm{In}_{8.75} \mathrm{Sn}_{1.25} \mathrm{~S}_{18.5}\right]^{8}$ & $2 \mathrm{D}$ & 0.1 & MB & $80,>90$ & NA & 0.5 \\
\hline
\end{tabular}

${ }^{\mathrm{a} F o r}$ convenience, only framework elements are presented. ${ }^{\mathrm{b}} \mathrm{MB}=$ Methylene Blue; $\mathrm{RhB}=$

Rhodamine B; CV = Crystal Violet

(2) Inorg. Chem. 2020, 59, 12, 7919-7923; (3) Cryst. Growth Des. 2017, 17, 2936-2940; (4) Inorg. Chem. 2017, 56, 14763-14766; (5) Inorg. Chem. 2016, 55, 10855-10858; (6) Chem. Commun. 2019, 55, 11083; (7) Dalton Trans. 2015, 44, 6400-6405; (8) Cryst. Growth Des. 2019, 19, 4151-4156.

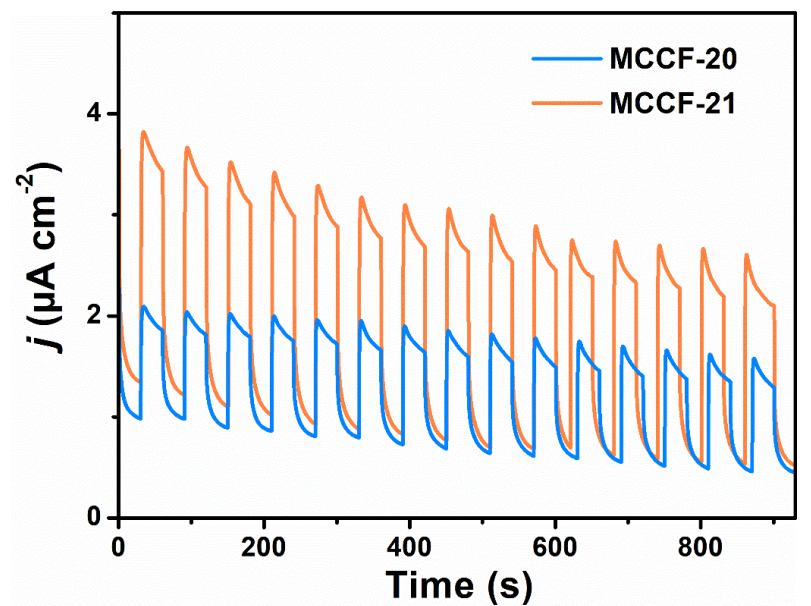

Figure S22. Photocurrent response curves of MCCF-20 and MCCF-21. 
Table S5. Bond Angles for MCCF-20 ${ }_{\mathrm{L}}$.

\begin{tabular}{|c|c|c|c|c|c|c|c|}
\hline Atom & Atom & Atom & Angle $/^{\circ}$ & Atom & Atom & Atom & Angle $/^{\circ}$ \\
\hline S1 & Sn1 & S3 & $113.31(8)$ & $\mathrm{S} 1^{1}$ & $\mathrm{Zn} 2$ & S6 & $106.71(9)$ \\
\hline S1 & Sn1 & S4 & $95.91(6)$ & $\mathrm{S} 1^{1}$ & $\mathrm{Zn} 2$ & S8 & $109.67(5)$ \\
\hline S2 & Sn1 & S1 & $113.74(7)$ & $\mathrm{S} 2^{3}$ & $\mathrm{Zn} 2$ & S6 & $107.17(7)$ \\
\hline S2 & Sn1 & $\mathrm{S} 3$ & $112.55(7)$ & S8 & $\mathrm{Zn} 2$ & $\mathrm{~S} 2^{3}$ & $112.09(6)$ \\
\hline S2 & Sn1 & S4 & $109.28(8)$ & S8 & $\mathrm{Zn} 2$ & S6 & $110.72(7)$ \\
\hline S3 & Sn1 & S4 & $110.81(7)$ & $\mathrm{Zn} 2^{4}$ & $\mathrm{~S} 1$ & Sn1 & $105.86(7)$ \\
\hline S5 & $\mathrm{Sn} 2$ & S4 & $109.73(6)$ & $\mathrm{Zn} 2^{5}$ & $\mathrm{~S} 2$ & Sn1 & $102.65(7)$ \\
\hline S5 & $\mathrm{Sn} 2$ & S6 & $111.82(8)$ & $\mathrm{Zn} 1^{4}$ & $\mathrm{~S} 3$ & Sn1 & $102.14(7)$ \\
\hline S5 & $\mathrm{Sn} 2$ & S7 & $112.72(8)$ & Sn2 & S4 & Sn1 & $109.37(7)$ \\
\hline S6 & $\mathrm{Sn} 2$ & S4 & $106.38(7)$ & $\mathrm{Zn} 1$ & S5 & $\mathrm{Sn} 2$ & $104.43(7)$ \\
\hline S7 & $\mathrm{Sn} 2$ & S4 & $101.15(7)$ & $\mathrm{Zn} 2$ & S6 & $\mathrm{Sn} 2$ & $103.99(7)$ \\
\hline S7 & $\mathrm{Sn} 2$ & S6 & $114.23(8)$ & $\mathrm{Zn} 1^{2}$ & S7 & $\mathrm{Sn} 2$ & $103.20(8)$ \\
\hline $\mathrm{S} 3^{1}$ & $\mathrm{Zn} 1$ & S5 & $106.28(7)$ & $\mathrm{Zn} 1^{2}$ & S8 & $\mathrm{Zn} 1$ & $110.23(10)$ \\
\hline $\mathrm{S} 3^{1}$ & $\mathrm{Zn} 1$ & $\mathrm{~S} 7^{2}$ & $109.30(7)$ & $\mathrm{Zn} 1$ & S8 & $\mathrm{Zn} 2$ & $110.59(2)$ \\
\hline $\mathrm{S} 3^{1}$ & $\mathrm{Zn} 1$ & S8 & $112.72(7)$ & $\mathrm{Zn} 1$ & S8 & $\mathrm{Zn} 2^{2}$ & $108.30(2)$ \\
\hline$S 7^{2}$ & $\mathrm{Zn} 1$ & S5 & $106.28(8)$ & $\mathrm{Zn} 1^{2}$ & S8 & $\mathrm{Zn} 2^{2}$ & $110.59(2)$ \\
\hline S8 & $\mathrm{Zn} 1$ & S5 & $110.42(5)$ & $\mathrm{Zn} 1^{2}$ & S8 & $\mathrm{Zn} 2$ & $108.30(2)$ \\
\hline S8 & $\mathrm{Zn} 1$ & $S 7^{2}$ & $111.51(6)$ & $\mathrm{Zn} 2$ & $\mathrm{~S} 8$ & $\mathrm{Zn} 2^{2}$ & $108.82(9)$ \\
\hline $\mathrm{S} 1^{1}$ & $\mathrm{Zn} 2$ & $\mathrm{~S} 2^{3}$ & $110.32(8)$ & & & & \\
\hline
\end{tabular}

${ }^{1} 1-Y,+X-Y, 1 / 3+Z ;{ }^{2}-Y+X,-Y, 5 / 3-Z ;{ }^{3} 1-X,-X+Y, 4 / 3-Z ;{ }^{4} 1+Y-X, 1-X,-1 / 3+Z ;{ }^{5} 1-X, 1-X+Y, 4 / 3-Z$

Table S6. Bond Lengths for MCCF-20 ${ }_{L}$.

\begin{tabular}{cccccc}
\hline Atom & Atom & Length/ $\mathbf{A}$ & Atom & Atom & Length/ $\AA$ \\
\hline Sn1 & $\mathrm{S} 1$ & $2.3754(17)$ & $\mathrm{Zn} 1$ & $\mathrm{~S} 3^{1}$ & $2.3422(19)$ \\
$\mathrm{Sn} 1$ & $\mathrm{~S} 2$ & $2.3583(19)$ & $\mathrm{Zn} 1$ & $\mathrm{~S} 5$ & $2.3523(18)$ \\
$\mathrm{Sn} 1$ & $\mathrm{~S} 3$ & $2.379(2)$ & $\mathrm{Zn} 1$ & $\mathrm{~S} 7^{2}$ & $2.3493(19)$ \\
$\mathrm{Sn} 1$ & $\mathrm{~S} 4$ & $2.4220(19)$ & $\mathrm{Zn} 1$ & $\mathrm{~S} 8$ & $2.3454(15)$ \\
$\mathrm{Sn} 2$ & $\mathrm{~S} 4$ & $2.4151(17)$ & $\mathrm{Zn} 2$ & $\mathrm{~S} 1^{1}$ & $2.3090(19)$ \\
$\mathrm{Sn} 2$ & $\mathrm{~S} 5$ & $2.3604(18)$ & $\mathrm{Zn} 2$ & $\mathrm{~S} 2^{3}$ & $2.3509(19)$ \\
$\mathrm{Sn} 2$ & $\mathrm{~S} 6$ & $2.393(2)$ & $\mathrm{Zn} 2$ & $\mathrm{~S} 6$ & $2.3524(19)$ \\
$\mathrm{Sn} 2$ & $\mathrm{~S} 7$ & $2.379(2)$ & $\mathrm{Zn} 2$ & $\mathrm{~S} 8$ & $2.3495(14)$ \\
\hline
\end{tabular}

${ }^{1} 1-Y,+X-Y, 1 / 3+Z ;{ }^{2}-Y+X,-Y, 5 / 3-Z ;{ }^{3} 1-X,-X+Y, 4 / 3-Z$ 
Table S7. Bond Angles for MCCF-21.

\begin{tabular}{|c|c|c|c|c|c|c|c|}
\hline Atom & Atom & Atom & Angle $/^{\circ}$ & Atom & Atom & Atom & Angle $/^{\circ}$ \\
\hline S3 & Sn1 & $\mathrm{S} 2$ & $110.76(7)$ & $\mathrm{S} 3$ & $\mathrm{Zn} 2$ & S11 & $108.29(7)$ \\
\hline $\mathrm{S} 3$ & Sn1 & S5 & $113.64(7)$ & $\mathrm{S} 10$ & $\mathrm{Zn} 2$ & S11 & $106.63(8)$ \\
\hline S4 & Sn1 & S2 & $107.16(7)$ & $\mathrm{S} 1$ & $\mathrm{Zn} 3$ & S4 & $110.95(6)$ \\
\hline $\mathrm{S} 4$ & Sn1 & S3 & $113.41(7)$ & S6 & $\mathrm{Zn} 3$ & $\mathrm{~S} 1$ & $111.86(6)$ \\
\hline S4 & Sn1 & S5 & $113.14(7)$ & S6 & $\mathrm{Zn} 3$ & S4 & $106.79(7)$ \\
\hline S5 & Sn1 & S2 & $97.37(6)$ & S7 & $\mathrm{Zn} 3$ & $\mathrm{~S} 1$ & $110.65(6)$ \\
\hline S7 & $\mathrm{Sn} 2$ & S14 & $113.95(7)$ & S7 & $\mathrm{Zn} 3$ & S4 & $108.56(7)$ \\
\hline S7 & $\mathrm{Sn} 2$ & S15 & $96.09(6)$ & S7 & $\mathrm{Zn} 3$ & S6 & $107.86(8)$ \\
\hline S8 & $\mathrm{Sn} 2$ & S7 & $113.81(7)$ & $\mathrm{S} 1$ & $\mathrm{Zn} 4$ & S13 & $111.10(6)$ \\
\hline S8 & $\mathrm{Sn} 2$ & S14 & $112.83(6)$ & $\mathrm{S} 1$ & $\mathrm{Zn} 4$ & S14 & $111.58(6)$ \\
\hline S8 & $\mathrm{Sn} 2$ & S15 & $108.55(7)$ & $\mathrm{S} 12$ & $\mathrm{Zn} 4$ & $\mathrm{~S} 1$ & $111.40(6)$ \\
\hline $\mathrm{S} 14$ & $\mathrm{Sn} 2$ & S15 & $110.22(7)$ & $\mathrm{S} 12$ & $\mathrm{Zn} 4$ & S13 & $107.51(7)$ \\
\hline S9 & Sn3 & $\mathrm{S} 13$ & $113.67(7)$ & $\mathrm{S} 12$ & $\mathrm{Zn} 4$ & S14 & $106.29(7)$ \\
\hline S9 & Sn3 & $\mathrm{S} 15^{1}$ & $100.60(7)$ & $\mathrm{S} 13$ & $\mathrm{Zn} 4$ & S14 & $108.75(7)$ \\
\hline $\mathrm{S} 10$ & $\mathrm{Sn} 3$ & S9 & $113.99(7)$ & $\mathrm{Zn} 1$ & $\mathrm{~S} 1$ & $\mathrm{Zn} 2$ & $109.47(6)$ \\
\hline $\mathrm{S} 10$ & Sn3 & $\mathrm{S} 13$ & $110.71(7)$ & $\mathrm{Zn} 1$ & $\mathrm{~S} 1$ & $\mathrm{Zn} 3$ & $109.62(6)$ \\
\hline $\mathrm{S} 10$ & Sn3 & $\mathrm{S} 15^{1}$ & $112.54(6)$ & $\mathrm{Zn} 1$ & $\mathrm{~S} 1$ & $\mathrm{Zn} 4$ & $109.74(6)$ \\
\hline $\mathrm{S} 13$ & $\mathrm{Sn} 3$ & $\mathrm{~S} 15^{1}$ & $104.56(7)$ & $\mathrm{Zn} 2$ & $\mathrm{~S} 1$ & $\mathrm{Zn} 3$ & $108.71(6)$ \\
\hline S6 & $\mathrm{Sn} 4$ & $\mathrm{~S} 2^{2}$ & $97.84(6)$ & $\mathrm{Zn} 2$ & $\mathrm{~S} 1$ & $\mathrm{Zn} 4$ & $109.54(6)$ \\
\hline S6 & $\mathrm{Sn} 4$ & $\mathrm{~S} 11$ & $112.15(7)$ & $\mathrm{Zn} 4$ & $\mathrm{~S} 1$ & $\mathrm{Zn} 3$ & $109.74(6)$ \\
\hline $\mathrm{S} 11$ & $\mathrm{Sn} 4$ & $\mathrm{~S} 2^{2}$ & $109.69(7)$ & Sn1 & $\mathrm{S} 2$ & $\mathrm{Sn} 4^{3}$ & $106.73(7)$ \\
\hline $\mathrm{S} 12$ & $\mathrm{Sn} 4$ & $\mathrm{~S} 2^{2}$ & $108.68(7)$ & $\mathrm{Zn} 2$ & S3 & Sn1 & $102.04(7)$ \\
\hline $\mathrm{S} 12$ & $\mathrm{Sn} 4$ & S6 & $113.36(7)$ & $\mathrm{Zn} 3$ & S4 & Sn1 & $103.83(7)$ \\
\hline $\mathrm{S} 12$ & $\mathrm{Sn} 4$ & $\mathrm{~S} 11$ & $113.86(7)$ & $\mathrm{Zn} 1$ & S5 & Sn1 & $103.24(7)$ \\
\hline $\mathrm{S} 1$ & $\mathrm{Zn} 1$ & S8 & $111.06(7)$ & $\mathrm{Zn} 3$ & S6 & $\mathrm{Sn} 4$ & $103.34(7)$ \\
\hline S1 & $\mathrm{Zn} 1$ & S9 & $110.94(6)$ & $\mathrm{Zn} 3$ & S7 & $\mathrm{Sn} 2$ & $103.73(7)$ \\
\hline S5 & $\mathrm{Zn} 1$ & $\mathrm{~S} 1$ & 111.91(6) & $\mathrm{Zn} 1$ & $\mathrm{~S} 8$ & $\mathrm{Sn} 2$ & $103.44(7)$ \\
\hline S5 & $\mathrm{Zn} 1$ & S8 & $107.88(8)$ & $\mathrm{Zn} 1$ & S9 & $\mathrm{Sn} 3$ & $104.32(7)$ \\
\hline S5 & $\mathrm{Zn} 1$ & S9 & $108.13(8)$ & $\mathrm{Zn} 2$ & $\mathrm{~S} 10$ & $\mathrm{Sn} 3$ & $104.09(7)$ \\
\hline S8 & $\mathrm{Zn} 1$ & S9 & $106.72(7)$ & $\mathrm{Zn} 2$ & $\mathrm{~S} 11$ & $\mathrm{Sn} 4$ & $103.23(6)$ \\
\hline $\mathrm{S} 1$ & $\mathrm{Zn} 2$ & S3 & $112.48(6)$ & $\mathrm{Zn} 4$ & $\mathrm{~S} 12$ & $\mathrm{Sn} 4$ & $103.93(7)$ \\
\hline $\mathrm{S} 1$ & $\mathrm{Zn} 2$ & $\mathrm{~S} 10$ & $111.03(6)$ & $\mathrm{Zn} 4$ & $\mathrm{~S} 13$ & $\mathrm{Sn} 3$ & $103.79(7)$ \\
\hline S1 & $\mathrm{Zn} 2$ & $\mathrm{~S} 11$ & $111.04(6)$ & $\mathrm{Zn} 4$ & S14 & $\mathrm{Sn} 2$ & $102.09(6)$ \\
\hline $\mathrm{S} 3$ & $\mathrm{Zn} 2$ & $\mathrm{~S} 10$ & 107.11(8) & $\mathrm{Sn} 2$ & S15 & $\mathrm{Sn} 3^{4}$ & $109.96(7)$ \\
\hline
\end{tabular}

${ }^{1} 1-\mathrm{X}, 1 / 2+\mathrm{Y}, 3 / 2-\mathrm{Z} ;{ }^{2} 1 / 2+\mathrm{X}, 3 / 2-\mathrm{Y}, 1-\mathrm{Z} ;{ }^{3}-1 / 2+\mathrm{X}, 3 / 2-\mathrm{Y}, 1-\mathrm{Z} ;{ }^{4} 1-\mathrm{X},-1 / 2+\mathrm{Y}, 3 / 2-\mathrm{Z}$ 
Table S8. Bond Lengths for MCCF-21.

\begin{tabular}{|c|c|c|c|c|c|}
\hline Atom & Atom & Length/Å & Atom & Atom & Length/Å \\
\hline $\mathrm{Sn} 1$ & S2 & $2.4249(17)$ & $\mathrm{Zn} 1$ & S1 & $2.3375(16)$ \\
\hline $\operatorname{Sn} 1$ & $\mathrm{~S} 3$ & $2.386(2)$ & $\mathrm{Zn} 1$ & S5 & $2.3329(19)$ \\
\hline Sn1 & S4 & $2.3561(19)$ & Zn1 & S8 & $2.3454(18)$ \\
\hline Sn1 & S5 & $2.3907(18)$ & Zn1 & S9 & $2.3505(18)$ \\
\hline $\operatorname{Sn} 2$ & S7 & $2.3771(17)$ & $\mathrm{Zn} 2$ & S1 & $2.3468(16)$ \\
\hline $\mathrm{Sn} 2$ & S8 & $2.358(2)$ & $\mathrm{Zn} 2$ & S3 & $2.3574(19)$ \\
\hline $\operatorname{Sn} 2$ & S14 & $2.3829(17)$ & $\mathrm{Zn} 2$ & $\mathrm{~S} 10$ & $2.3580(18)$ \\
\hline $\operatorname{Sn} 2$ & S15 & $2.4370(16)$ & $\mathrm{Zn} 2$ & S11 & $2.3790(18)$ \\
\hline $\operatorname{Sn} 3$ & S9 & $2.3722(19)$ & $\mathrm{Zn} 3$ & S1 & $2.3488(16)$ \\
\hline $\operatorname{Sn} 3$ & S10 & $2.3666(17)$ & $\mathrm{Zn} 3$ & S4 & $2.3512(18)$ \\
\hline $\operatorname{Sn} 3$ & S13 & $2.3838(19)$ & $\mathrm{Zn} 3$ & S6 & $2.3428(18)$ \\
\hline $\operatorname{Sn} 3$ & S151 & $2.4372(17)$ & $\mathrm{Zn} 3$ & S7 & $2.3395(18)$ \\
\hline Sn 4 & S22 & $2.4363(18)$ & Zn4 & S1 & $2.3478(15)$ \\
\hline $\mathrm{Sn} 4$ & S6 & $2.3797(18)$ & $\mathrm{Zn} 4$ & $\mathrm{~S} 12$ & $2.3394(19)$ \\
\hline $\mathrm{Sn} 4$ & S11 & $2.3856(19)$ & $\mathrm{Zn} 4$ & $\mathrm{~S} 13$ & $2.3555(18)$ \\
\hline Sn4 & $\mathrm{S} 12$ & $2.3663(18)$ & $\mathrm{Zn} 4$ & S14 & $2.3675(17)$ \\
\hline
\end{tabular}

${ }^{1} 1-\mathrm{X}, 1 / 2+\mathrm{Y}, 3 / 2-\mathrm{Z} ;{ }^{2} 1 / 2+\mathrm{X}, 3 / 2-\mathrm{Y}, 1-\mathrm{Z}$ 